\title{
Project evaluation and cash flow forecasting by stochastic simulation
}

\author{
ODD ANDREAS ASBJØRNSEN
}

Keywords: stochastic, cash flow, simulation, project evaluation, optimal design.

The net present value of a discounted cash flow is used to evaluate projects. It is shown that the LaPlace transform of the cash flow time function is particularly useful when the cash flow profiles may be approximately described by ordinary linear differential equations in time. However, real cash flows are stochastic variables due to the stochastic nature of the disturbances during production.

Two approaches to the analysis and solution of such problems are demonstrated. The first considers averages over an infinite event space with specified probability distributions, and the second considers Monte Carlo simulations with a limited number of events occurring in a lifetime. Very interesting solutions to the problems of optimal plant capacity and product intermediate storage capacity are shown.

\section{Introduction}

At the initial stage of project evaluation and decision, one is often faced with the problem associated with uncertain assumptions related to the future cash flows. Most of these uncertainties are related to the market and price situations, but the uncertainties to productivity, reliability and availability of the production plants and distribution systems are sometimes of great significance. This is the situation particularly in offshore production where one often is working with designs at the edge of known and proved technology. The uncertainty in technical and operational assumptions becomes significant.

A better insight into the consequence of some of the basic assumptions for the design may be obtained by simulation. Such simulations will be of the Monte Carlo type, where random events in the future like shutdowns, market fluctuations, transportation irregularities, etc. are drawn from distributions. The purpose of this paper is to draw the attention to some applications which may be arrived at with very simple means, and where the main feature of a systems behaviour may be viewed from grossly simplified models.

\section{Net present value, continuous discount rate}

In order to evaluate and compare future projects at present, an estimate of the possible future profile of the net cash flow is needed. If this time function is established, the present value of all future cash flows may be accumulated in the net present value (NPV) of the project. Future cash flows are given a weight or significance which decreases with distance into the future, the so-called discount factor. The

Received 10 August 1983.

† University of Houston, Department of Chemical Engineering, Houston, TX 77004, U.S.A. 
discount factor is normally calculated per day, month or year, but in the present analysis, an exponential weighting factor is most useful (Asbjørnsen and Kreyberg 1979 , Johns and Vardas 1979). This is equivalent to applying a continuous interest rate.

If one considers a distance $T$ into the future (usually the project lifetime), then the accumulated net present value of the future net cash flow is

$$
\mathrm{NPV}=\int_{0}^{T} c(t) \exp (-r) d t
$$

or

$$
\mathrm{NPV}=\int_{0}^{\infty} c(t) \exp (-r t) d t ; \quad c(t)=0 \text { for } T<t
$$

The last formulation is identical to the definition of the Laplace transform (Churchill 1958), where the cash flow is set to zero after the project lifetime, and the Laplace variable $s$ is replaced by the discount rate

$$
\mathrm{NPV}=C(r)
$$

One may therefore conclude, as shown for example by Tanchucho et al. (1981) and Buck and Hill (1971).

If an artificial project lifetime is taken to be infinity, then the net present value is the Laplace transform of the net cash flow time function with the complex argument replaced by the discount rate. For a real and finite lifetime, the net cash flow is set to zero after a delay equal to the real lifetime of the project.

Take as an example a typical cash flow profile as illustrated in Fig. 1. Such a cash flow profile may be considered as the result of two competing mechanisms,

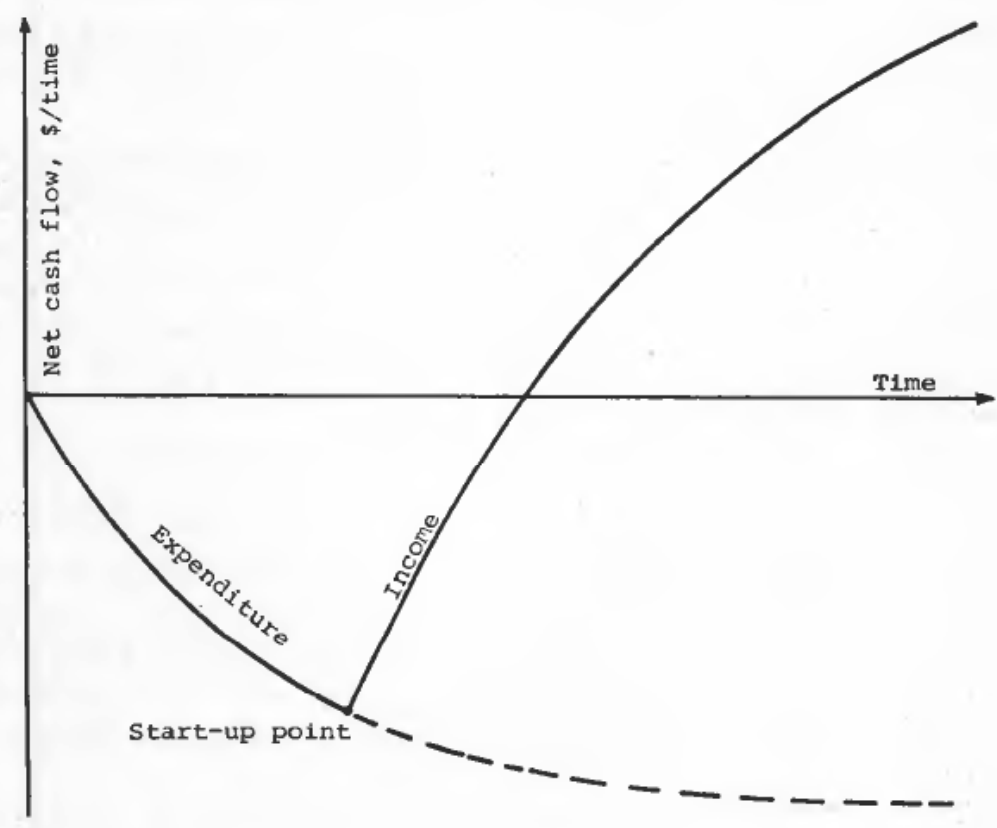

Figure 1. Typical cash flow profile for a project. 
the positive cash flow from production gains competing against the negative cash flow from investment and building cost expenditures. Both mechanisms may be modelled for convenience by first order differential equations. The rate of expenditure is assumed to be proportional to the difference between the final and actual investment

$$
\text { Rate of expenditure } c_{t}=\frac{d I}{d t}=k_{i}\left(I_{\infty}-I\right) ; 0 \leqslant t
$$

The cash flow from the production is coming after the start-up at a later stage and it is assumed that the rate of change of this cash flow is proportional to the difference between the final and actual cash flow rate. This is a model which describes an asymptotic development of the productivity towards an ultimate degree of perfection analogous to the learning curve

$$
\frac{d c_{p}}{d t}=k_{p}\left(c_{\infty}-c_{p}\right) ; \quad T_{0} \leqslant t
$$

This net cash flow rate covering the investment profile as well as the production profile may then be approximated by the simple difference

$$
c_{n}=c_{p}-c_{i}
$$

Since the two terms in the net cash flow are modelled by linear differential equations, the net present value is easily computed by the Laplace transform of the equations, provided an infinite project lifetime is assumed

$$
C_{n}(s)=\frac{k_{p} c_{\infty}}{s\left(s+k_{p}\right)} \exp \left(-s T_{0}\right)-\frac{k_{i} I_{\infty}}{s+k_{i}}
$$

Replacing the Laplace variable with the discount rate, the net present value of this project is

$$
\mathrm{NPV}=\frac{k_{p} c_{\infty} \exp \left(-r T_{0}\right)}{r\left(r+k_{p}\right)}-\frac{k_{i} I_{\infty}}{r+k_{i}}
$$

If one assumes a discount rate of $r=0.15$ year $^{-1}$, a time constant of investment of $1 / k_{i}=1$ year, a time constant for the learning curve of $1 / k_{p}=1$ year, a start-up time after $T_{0}=3$ years and a net return on the investment of $30 \%$, i.e. $c_{\infty} / I_{\infty}=0 \cdot 3$, then the net present value is computed to be about $24 \%$ of the total investment.

This example shows that it is useful to apply the Laplace transformation to the cash flow and the net present value, in particular if the cash flow profile may be modelled by differential equations.

A sensitivity analysis as to the various parameters like start-up time, discount rate, rate of return on investment and time constants in the cash flow model is particularly simple to obtain from the Laplace transform. Take the example above as an illustration.

Then the sensitivity in the net present value to changes in various parameters is easily obtained from the Laplace transform in eqn. (7) above. With the numerical data suggested, it is practical to normalize the NPV by dividing through with the total investment $I_{\infty}$. Let $V=\mathrm{NPV} / I_{\infty}$ and let $R=c_{\infty} / I_{\infty}$ be the net return on the total 
investment. Then the following sensitivities are computed

$$
\begin{aligned}
& r \frac{\partial V}{\partial r}=1 \cdot 64 ; \quad T_{0} \frac{\partial V}{\partial T_{0}}=-0 \cdot 5 \\
& R \frac{\partial V}{\partial R}=1 \cdot 11 ; \quad k_{p} \frac{\partial V}{\partial k_{p}}=0 \cdot 145 \\
& k_{i} \frac{\partial V}{\partial k_{i}}=-0.113
\end{aligned}
$$

It is seen that the greatest relative sensitivity in the NPV is found in the discount rate. If the relative change in $r$ is an increase of $5 \%$ (i.e. $r$ increases from 15 to $15.75 \%$ per year), the normalized $\operatorname{NPV}(V)$ decreases with $8.2 \%$ of the total investment. The second greatest sensitivity is in the net return on total investment $R$. If this increases with $5 \%$ (i.e. from 0.3 to 0.315 ) then the normalized NPV increases with $5.6 \%$. Similarly, if the start-up time is delayed by $5 \%$ (i.e. by 54 days) the normalized NPV is reduced with $2 \cdot 5 \%$ (i.e. by $2.5 \%$ of the total investment).

The sensitivities in the rate constant of the 'learning curve' and in the rate constant of the investment are much lower, roughly one tenth of the sensitivity in the discount rate or the net return on investment. These two parameters are typically production technology oriented, while the previous parameters are related to over-all economic policy and project management. It is in these areas one finds the most pronounced effects on the feasibility of a project.

Even if this example is greatly simplified, it demonstrates the effects of some of the crucial parameters and variables in the economy of a project. The cash flow profile is essential and reasonable models for this profile are required, especially in the initial stage of project evaluations. As emphasized by Asbjørnsen and Kreyberg (1979), all aspects of process technology and chemical engineering have influence on this profile. The systems approach to mathematical modelling and computer simulations lead to an accumulation of knowledge, experience and knowhow which is essential for any chemical company in the competitive market with ever increasing constraints and restrictions on the design and operation.

\section{The maximum rate of return of discounted cash flow}

The discount rate which makes the net present value equal to zero is often referred to as the maximum rate of return of the discounted cash flow. Then the maximum rate of return is simply the zero of the Laplace transform of the cash flow rate, if for simplicity one again assumes an artificial infinite lifetime of the project

$$
C(r)=0
$$

Take the example in eqn. (7). Here, there is one zero given by the transcendental equation

$$
\frac{k_{p}}{k_{i}} \frac{c_{\infty}}{I_{\infty}}\left(r+k_{i}\right) \exp \left(-r T_{0}\right)-r\left(r+k_{p}\right)=0
$$

which gives the single root $r=17 \cdot 66 \%$ with the same data as used throughout.

Again, the example favours the use of the Laplace transform, as it gives simple and transparent expressions. Furthermore, any discussion about multiple roots introduced by the power series of a discontinuous annuity is avoided. 


\section{The pay back time}

For a given discount rate $r$, the time required for the present value of the net income cash flow to equal the present value of the total investment is often referred to as the pay back time. Although the Laplace transform in eqn. (3) may not be used directly the pay back time may be computed indirectly for the example considered by

$$
\int_{T_{0}}^{T} c_{p}\left(t-T_{0}\right) \exp (-r t) d t-\int_{0}^{T} c_{i}(t) \exp (-r t) d t=0
$$

or

$$
\exp \left(-r T_{0}\right) \int_{0}^{T-T_{0}} c_{p}(t) \exp (-r t) d t-\int_{0}^{T} c_{l}(t) \exp (-r t) d t=0
$$

In the example shown, the integrals may be derived from the Laplace transforms, and one gets a semi-implicit equation

$$
\begin{aligned}
T-T_{0}=\frac{1}{r} \ln \left(\frac{k_{p}}{r+k_{p}}+\frac{r}{r+k_{p}}\right. & \exp \left(-\left(r+k_{p}\right)\left(T-T_{0}\right)\right) \\
& \left.-\frac{I_{\infty}}{c_{\infty}} \exp \left(r T_{0}\right) \frac{k_{i} r}{k_{i}+r}\left(1-\exp \left(-\left(r+k_{i}\right) T\right)\right)\right)
\end{aligned}
$$

After only two direct iterations, the error in the computed pay back time is less than $5 \times 10^{-4}$ years, and the solution is: $T=14 \cdot 15$ years. Hence, the time required to recover the total investment is about 11 years from the start of production.

If the interest rate is reduced to $7 \%$ then the pay back time is reduced considerably, and calculated to be $T=8.42$ years, i.e. 5.42 years after start of production.

One may twist the problem somewhat, and ask what the required net rate of return on investment $c_{\infty} / I_{\infty}$ has to be in order for the pay back time to be 5 years from the start of production at an interest rate of $15 \%$. The answer is

$$
\frac{c_{\infty}}{I_{\infty}}=\frac{k_{i}\left(r+k_{p}\right)\left(1-\exp \left(-\left(r+k_{i}\right) T\right) r\right.}{\left(r+k_{i}\right)\left(k_{p} \exp \left(-r T_{0}\right)+\left(r \exp \left(k_{p}\left(T_{0}-T\right)\right)-\left(r+k_{p}\right)\right) \exp (-r T)\right)}=0.514
$$

This shows that the original rate of return on investment should be raised by about $70 \%$ to recover the investment in five years at an interest rate of $15 \%$ on the financial market.

\section{The inflation problem}

Inflation is a more or less permanent phenomenon in the post war economic development, and it is required to take this into account. Inflation means that the cash flows in actual monetary units increase duc to price increase. If one takes the product of quantity and price, then the cash flow will increase proportional to the price

$$
c(t)=q(t) p(t)
$$

A frequently used model for the price development is an exponential growth ratc of a price index

$$
p(t)=p_{0} \exp \left(i\left(t-t_{0}\right)\right)
$$


where $p_{0}$ is a reference price or a price index at time $t_{0}$. The inflation rate $i$ is seen to work against the interest rate, as the discounted cash flow may be written

$$
\begin{aligned}
\mathrm{NPV}=\int_{0}^{T} c(t) \exp (-r t) d t=\int_{0}^{T} q(t) p(t) \exp (-r t) d t \\
=p_{0} \int_{0}^{T} q(t) \exp (-(r-i) t) d t
\end{aligned}
$$

where the reference price is taken at $t_{0}=0$. The difference between the discount rate $r$ and the inflation rate is sometimes referred to as the real interest rate. The same models as used before apply to investment analysis under inflation too, as long as the real interest rate is used.

The inflation imposes an extra problem by the fact that the inflation rates may be different from one item to another in the net cash flow sum. The modelling of this phenomenon is straight forward in principle, but good estimates of real and reliable data for the inflation and interest rates, not to mention step changes in prices, are hard to find. This makes the foundation of the modelling of investment analysis somewhat shaky. Still however, models are useful and simulation even more valuable to eludicate effects and mechanisms. The most crucial requirement is that the analyst knows what he is doing, and is able to interpret the simulation results in the light of the assumptions made.

\section{Discontinuous cash flow profiles}

In most practical cases the cash flow profile is not a continuous function of time. This applies to production plants with shut-downs and to most building and investment programmes. Then the cash flow curves may undergo step changes or impulse changes (dirac pulses). Investments are typically impulses while shutdowns of the production are step changes. The effect of impulses and step changes is usually attenuated by cumulative or inertia effects in the production or in the organization, analogous to low-pass filter characteristics in process dynamics, signal processing and communication theory.

A time series of impulses or step changes are easily modelled in the Laplace domain, as one has for step changes

$$
C(r)=\sum_{i=1}^{n} \exp \left(-r T_{i}\right) c_{i}(1-\exp (-r \Delta T)) / r
$$

where $\Delta T_{i}$ is the duration of the step change occurring at $T_{i}$. A train of impulses (dirac pulses) are modelled by

$$
C(r)=\sum_{i=1}^{n} \exp \left(-r T_{i}\right) c_{i}
$$

which is typical for discrete investments at time $T_{i}$.

These functions may hence enter any of the computational formulae shown for the net present value, maximum rate of return on investment, pay back time, etc.

\section{Project evaluation based on long-term average cash flow}

Cash flow estimates from a production are normally based on a set of average production rates or a profile of production rates as shown by the previous example. Such average production rates may not be applicable if there are frequent changes in 
the operating conditions like supply and demand changes, start-ups and shut-downs, etc. A more realistic approach to the description of such events may use probability distribution functions. A simple illustration is shown below. The illustration is applicable to a gas processing plant in an offshore field.

\section{Optimal plant capacity under varying supply and demand}

The supply of raw materials to the plant in Fig. 2 is assumed to vary over a certain range. Such variations may be described by a probability law characterized by an average and a standard deviation (swing factor). The plant capacity should match such conditions, but it is clearly not optimal to design the plant according to the maximum supply, because this is a situation which happens very rarely.

The demand may be described in a similar way, it also is assumed to vary over a certain range. This situation may again be modelled by a probability law and one is interested in the average net cash flow from the production and the optimal plant investment. A typical but grossly simplified picture is given in Fig. 2. Let the probability density functions be

$$
\left.\begin{array}{c}
\text { Supply }=\phi_{\mathrm{s}}(x) \\
\text { Demand }=\phi_{\mathrm{d}}(y)
\end{array}\right\}
$$

Assume the stochastic fluctuations in supply and demand to be uncorrelated. Then the probability for a supply between $x$ and $x+d x$ is $\phi_{s}(x) d x$ and similarly for demand between $y$ and $y+d y, \phi_{\mathrm{d}}(y) d y$. The plant capacity is $P^{*}$.

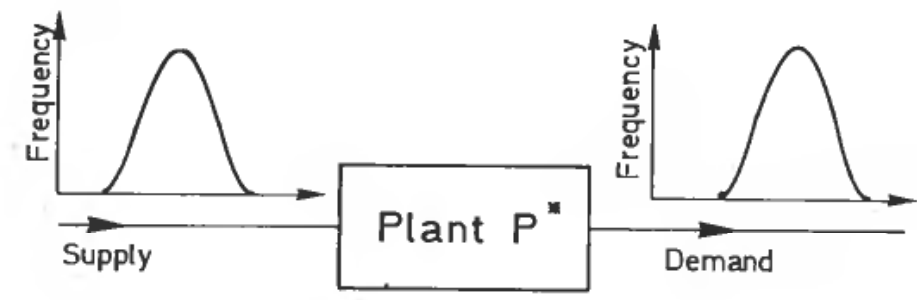

Figure 2. Total plant with varying supply and demand.

If the demand is $y$, then the market will be unsaturated if the supply $x<y$ or the plant design capacity $P^{*}<y$. The market will be flooded when the supply $x>y$ and simultaneously $P^{*}>x$. The probability that the supply is $x$ and at the same time the demand $y$ greater than the supply is

$$
d S_{x}=\left(1-F_{\mathrm{d}}(x)\right) \phi_{\mathrm{s}}(x) d x ; y>x
$$

Similarly, the probability that the demand is $y$ and at the same time the supply $x$ greater than the demand is

$$
d S_{y}=\left(1-F_{\mathrm{s}}(y)\right) \phi_{\mathrm{d}}(y) d y ; \quad y<x
$$

The probability that the supply $x$ is greater than the plant production capacity $P^{*}$ and at the same time the demand is greater than $P^{*}$ is

$$
S_{P^{*}}=\left(1-F_{\mathrm{s}}\left(P^{*}\right)\right)\left(1-F_{\mathrm{d}}\left(P^{*}\right)\right) ; \quad y>P^{*}, x>P^{*}
$$


The production rate is always adjusted to match the minimum (bottle neck) of the three variables, the supply $x$, the demand $y$ and the plant capacity $P^{*}$

$$
P=\min \left(x, y, P^{*}\right)
$$

Hence, the average production rate over a long period of time (theoretically speaking infinite time) is

$$
\begin{aligned}
\bar{P}=\int_{x_{\operatorname{man}}}^{P^{*}} x\left(1-F_{\mathrm{d}}(x)\right) \phi_{\mathrm{s}}(x) d x+\int_{y_{\min }}^{P^{*}} y\left(1-F_{\mathrm{s}}(y)\right) \phi_{\mathrm{d}}(y) d y \\
+P^{*}\left(1-F_{\mathrm{d}}\left(P^{*}\right)\right)\left(1-F_{\mathrm{s}}\left(P^{*}\right)\right)
\end{aligned}
$$

where $F_{\mathrm{d}}$ and $F_{\mathrm{s}}$ are the distribution functions of demand and supply, respectively. The economic objective function is taken to be the net present value of the discounted cash flow, and the cash flow is computed as the net gain from the average production and the plant investment, provided the market price and proportional costs remain constant. The plant investment is assumed to be proportional to the $\beta$-power of the capacity and to occur now

$$
\mathrm{NPV}=\bar{P} \cdot c \cdot\left(1-\exp \left(-T_{0} r\right)\right) / r-I_{0}\left(P^{*} / P_{0}\right)^{\beta}
$$

The optimal plant design capacity is that value which makes $\partial \mathrm{NPV} / \partial P^{*}=0$. Hence,

$$
P_{0} c / \exp \left(1-\exp \left(-T_{0} r\right)\right) / r \frac{\partial \bar{P}}{\partial P^{*}}-\beta I_{0}\left(P^{*} / P_{0}\right)^{\beta-1}=0
$$

where

$$
\frac{\partial \bar{P}}{\partial P^{*}}=\left(1-F_{\mathrm{d}}\left(P^{*}\right)\right)\left(1-F_{\mathrm{s}}\left(P^{*}\right)\right)
$$

The necessary condition for the simple analysis above to be applicable, is that the supply and demand are uncorrelated. Furthermore, that the price parameter $c$ remains independent of the market and supply situation, an assumption which may be justified under long term contracts.

Even if the investment and cash flow models are grossly simplified, an illustration may be of interest at this stage. Let the probability density function for supply and demand be triangular and symmetrical with a swing of $20 \%$. Let the rate of return $P_{0} c / I_{0}=0 \cdot 3$, the real interest rate $r=0 \cdot 15$ and the project lifetime $T_{0}=10$ years. Then the density functions are:

$$
\begin{aligned}
& P_{0} \phi_{\mathrm{s}}(x)=\frac{x-0.8}{0.04}=P_{0} \phi_{\mathrm{d}}(x) ; \quad 0.8<x<1 \\
& P_{\mathrm{o}} \phi_{\mathrm{s}}(x)=\frac{1 \cdot 2-x}{0.04}=P_{\mathrm{o}} \phi_{\mathrm{d}}(x) ; \quad 1.0<x<1.2
\end{aligned}
$$

and the distribution functions

$$
\begin{array}{ll}
F_{\mathrm{s}}(x)=\frac{(x-0 \cdot 8)^{2}}{0 \cdot 08}=F_{\mathrm{d}}(x) ; & 0 \cdot 8<x<1 \\
F_{\mathrm{s}}(x)=1-\frac{(1 \cdot 2-x)^{2}}{0 \cdot 08}=F_{\mathrm{d}}(x) ; & 1 \cdot 0<x<1 \cdot 2
\end{array}
$$


Although this is a completely artificial example, the optimal conditions for the plant capacity, as defined in eqns. (27) and (28), may be used to illustrate the relationship between investment, real interest rate, net annual cash flow and plant size at optimum in a simple way. Assuming triangular probability density functions as indicated, the optimal plant capacity is determined by the two equations below

$$
\begin{array}{r}
x^{0.3}\left(1-\frac{(x-0.8)^{2}}{0.08}\right)^{2}-\alpha=0 ; \quad 0.8 \leqslant x \leqslant 1 \\
x^{0.3}\left(\frac{1 \cdot 2-x}{0.08}\right)^{4}-\alpha=0 ; \quad 1 \leqslant x \leqslant 1.2
\end{array}
$$

where all parameters related to economics are grouped in $\alpha$

$$
\alpha=\beta I_{0} r /\left(c P_{0}\left(1-\exp \left(-T_{0} r\right)\right)\right)
$$

The optimal plant size as a function of this parameter for the triangular distributions chosen, is shown in Fig. 3. Note that there is an upper limit to $\alpha$ above which a plant is not feasible (an extremum on the curve in the direction of the abscissa). Above this limit, the investment is too high to be carried by the annual cash flow $c P_{0}$ at the real interest rate $r$. The optimal plant at this extremum has a capacity which fits the lowest level of the swing in supply and demand.

Considering the data chosen for the present illustration and letting $\beta=0 \cdot 7$, the value for $\alpha$ is

$$
\alpha=0 \cdot 7 * 0 \cdot 15 /(0 \cdot 3 *(1-\exp (-1 \cdot 5)))=0 \cdot 4505
$$

and the optimal capacity of the plant with a swing of $20 \%$ is about $96 \%$ of the average supply, according to Fig. 3 . This means that the average plant production utilizes only $68.65 \%$ of the average available supply.

It should be emphasized here, that the method of integration of the probability laws assumes an infinite number of events within the probability density functions, as these are considered continuous functions. In real life, the history of the plant and the supply and market situation is composed of a limited number of stochastic events. This leaves all derived properties like cash flow, optimal plant capacity, etc. to be stochastic properties with their average values and distribution functions. These aspects are demonstrated by a more elaborate model for stochastic simulation below.

\section{Project evaluation based on Monte Carlo simulation of cash flow}

An improved estimation of real cash flow time functions over a project lifetime may be obtained by stochastic simulation or Monte Carlo simulation. In this approach events are drawn from random distributions. Such events may include equipment which fails, raw material supply which fails, electricity supply which falls out, etc. Such sudden shocks cause interruption of the material flow, which has consequences for the economics of production as well as the material balances. The normal approach to analyse phenomena of this nature is by stochastic simulation or Monte Carlo simulation.

The unexpected shocks arc initiatcd from random number generators, where certain accepted probability density functions are applied. Since there is a very close relationship between material balances and cash flow, the stochastic simulation of random shocks over a time period is extended up to the lifetime of the project and the cash flow is computed from the material flow. From the stochastic cash flow, one may determine a more realistic picture of the real present value of the production. 


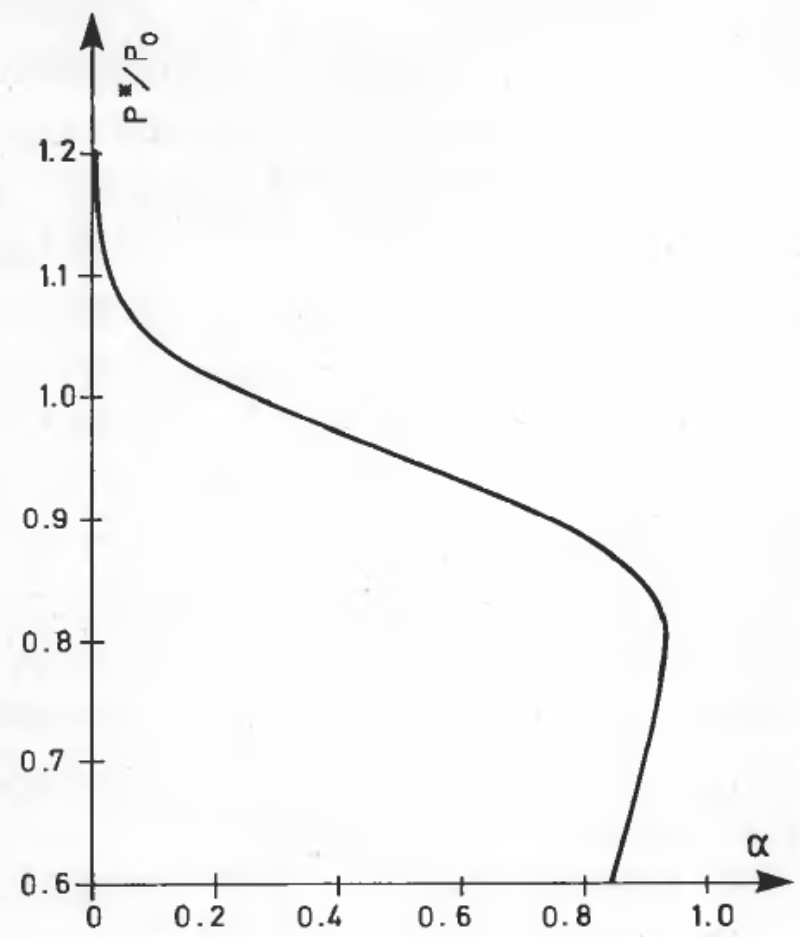

Figure 3. Optimal plant overdesign as function of the investment and yearly net cash flow ratio.

When a number of simulations are carried out, one may also get a picture of the probability density function and the variance of the present value. This variance is caused by the unreliability of the production, and will always cause the present value to be lower than the upper theoretical boundary which was computed from an assumption of $100 \%$ reliable plant and market.

Take as an example, a production and a shipping system with an intermediate storage, as shown in Fig. 4.

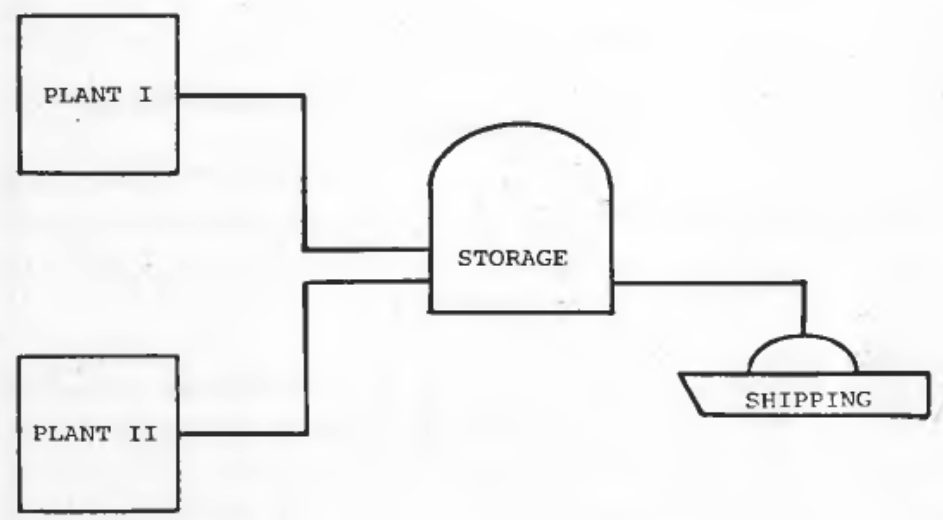

Figure 4. Production and shipping with intermediate storage. 
The production plant has two parallel equal units such that the material flow into the storage is

$$
w_{1}=\sum_{i=1}^{2} p_{i} \cdot \delta_{i}
$$

where $\delta_{i}=0$ means fall out of unit no. $i$ and $\delta_{l}=1$ means full production. This variable is a stochastic variable.

The shipping plant receive ships with a given load capacity $Q_{i}$, and this capacity is also considered a random variable. The arrival of ships follows a shipping plan, but unexpected disturbances may change this. Hence, the time of arrival of the ship is again a random variable. The loading capacity $w_{l i}$ of the ship is also taken as a random variable.

The material balance for the shipping section envisages a discontinuous flow such that the outflow from the storage is equal to the ship loading capacity during the time of loading

$$
w_{2}=w_{l i} \text { for } T_{i} \leqslant t \leqslant T_{i}+Q_{l} / w_{l i}
$$

The material balance for the storage is simply

$$
Q_{s}=\int_{0}^{t}\left(w_{1}-w_{2}\right) d t
$$

If it happens that the storage becomes empty during loading, then the ship has to leave without being completely loaded, at the time when the storage became empty. This situation is punished by a fine which is proportional to the difference between the desired and actual load attained. If the storage becomes full during production, then the plants are shut off and $w_{1}=0$. Since the loading pump capacity is higher than the production rate, this situation occurs only when the ship is absent, i.e. $w_{2}=0$ also.

The problem is described by five stochastic time events, the shut-down of any of the two parallel plants, their subsequent start-up and the arrival of ships. When the ship arrives, two random numbers are drawn, the ship size and the loading capacity.

Let the data in the Table specify the operating conditions.

\begin{tabular}{lccccl}
\hline Event & Max & Norm & Min & Unit & Distribution \\
\hline Shipload capacity & 20000 & 18000 & 16000 & $t$ & Triangular \\
Shiploading capacity & 10000 & 9000 & 8000 & $t / d$ & Triangular \\
Time between ship arrivals & $12 \cdot 6$ & $10 \cdot 6$ & $8 \cdot 6$ & $d$ & Triangular \\
Mean time to repair & 7 & 4 & 1 & $d$ & Triangular \\
Mean time between failure & & 20 & & $d$ & Exponential \\
Storage capacity & & 50000 & & $t$ & \\
Production & & 2000 & & $t / d$ & \\
\hline
\end{tabular}

Production and transport data.

The cash flow function for the operation may be taken as the sum of a positive term which is the sales value of the ship load, and some negative terms which are the proportional cost of the production, the fixed cost of production and the cost of 


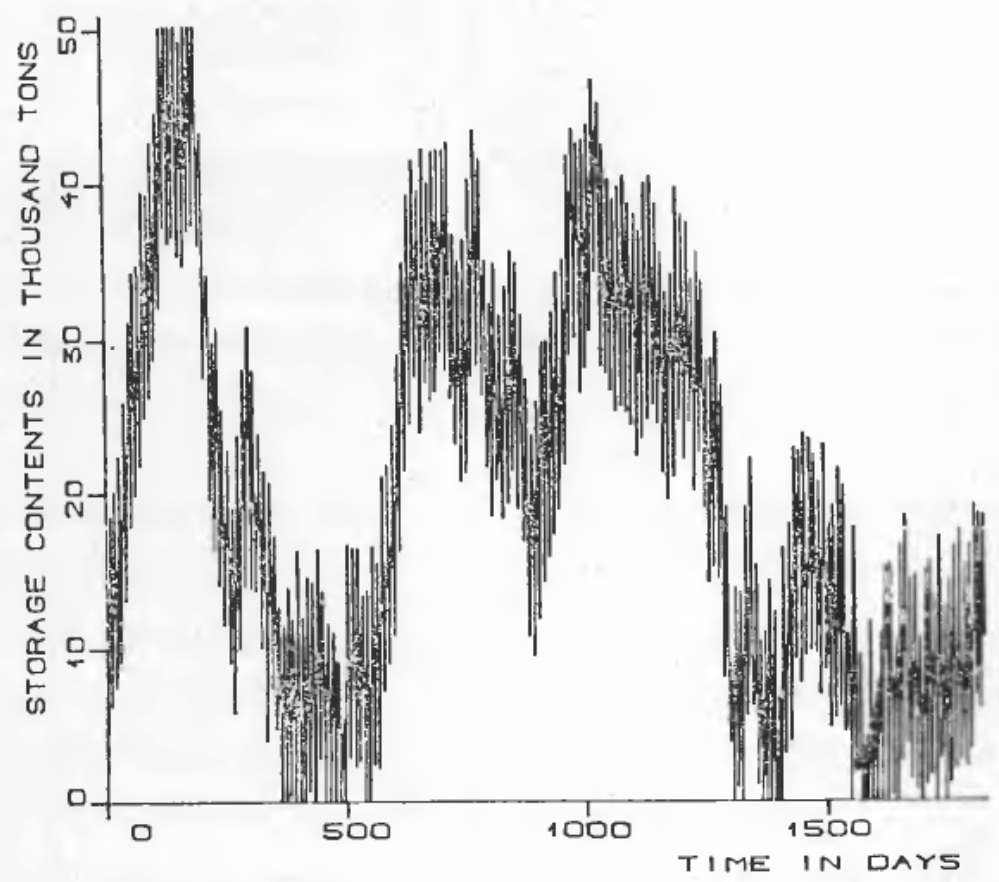

Figure 5. Storage contents over the years, uncontrolled shipping programme.

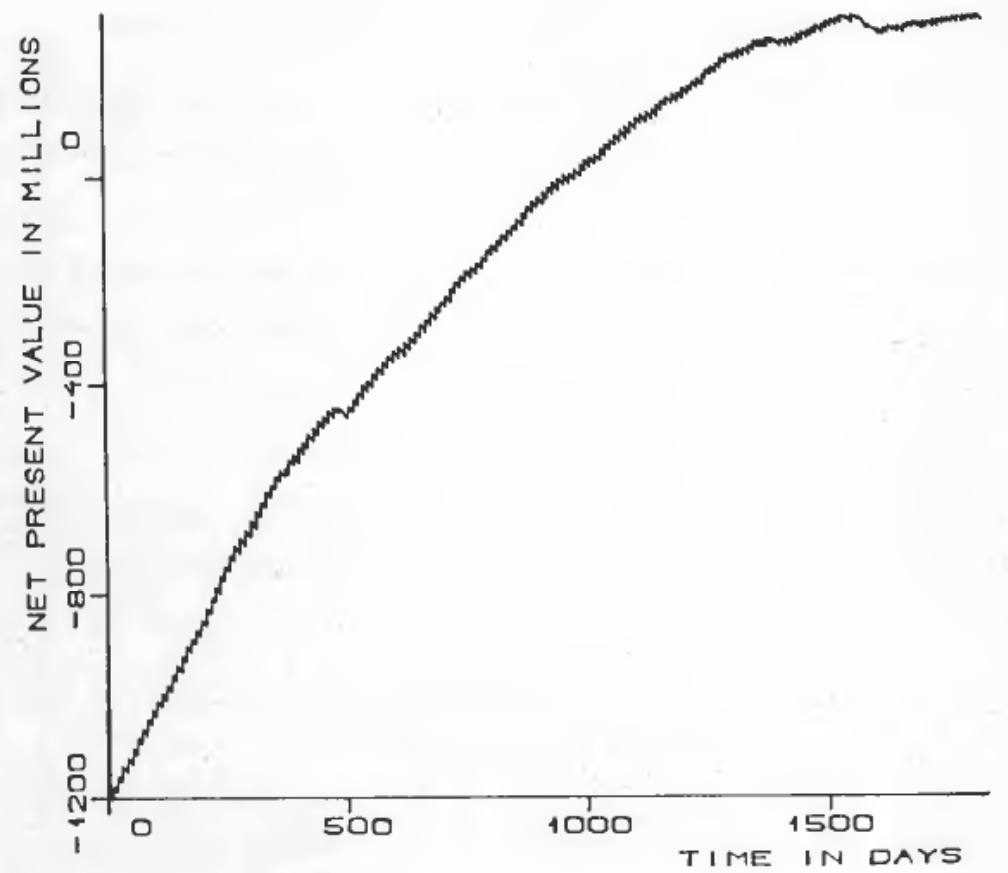

Figure 6. The accumulated discounted cash flow of the invested capital and production. 
eventual fines if the ship will not be completely loaded according to the specifications due to shut-downs of the plants and an eventual empty storage

$$
c(t)=\left(c_{s} Q_{i}-c_{d}\left(Q_{0 i}-Q_{i}\right)\right) \delta\left(T_{i}+\Delta T_{i}\right)-c_{p} w_{1}-c_{f}
$$

Here $c_{d}$ is the penalty factor which comes into account if by any chance the storage becomes empty during loading, and the ship is not completely loaded. This penalty expresses an expense related to the difference in ship load required $Q_{0}$ and the actual ship load $Q_{i}$ acquired due to the emptying of the storage.

The function $\delta\left(T_{t}+\Delta T_{i}\right)$ is a dirac impulse which signifies that the cash flow of the value of the ship load enters at the time of the departure of the ship. If required, a credit delay may be introduced at this point.

The first experiment which may be done with this very simple stochastic model of the production, the off-site storage farm and the shipping programme, is to elucidate and illustrate the history of the plants, their storage and the shipment.

A five year period is selected for illustration, and the storage contents and the net present value of the discounted cash flow over this period are shown in Figs. 5 and 6. As it is seen, the storage capacity is certainly fluctuating both regularly, due to the regular arrival and departure of the ships, and irregularly due to the irregular stochastic variables describing the arrival of the ships, the load and the loading capacity of the ships and the shut-downs and the start-ups of the plant. Furthermore, the tendency in the storage contents over the years is slowly decreasing. This is due to the fact that the average transport flow is somewhat greater than the average production flow.

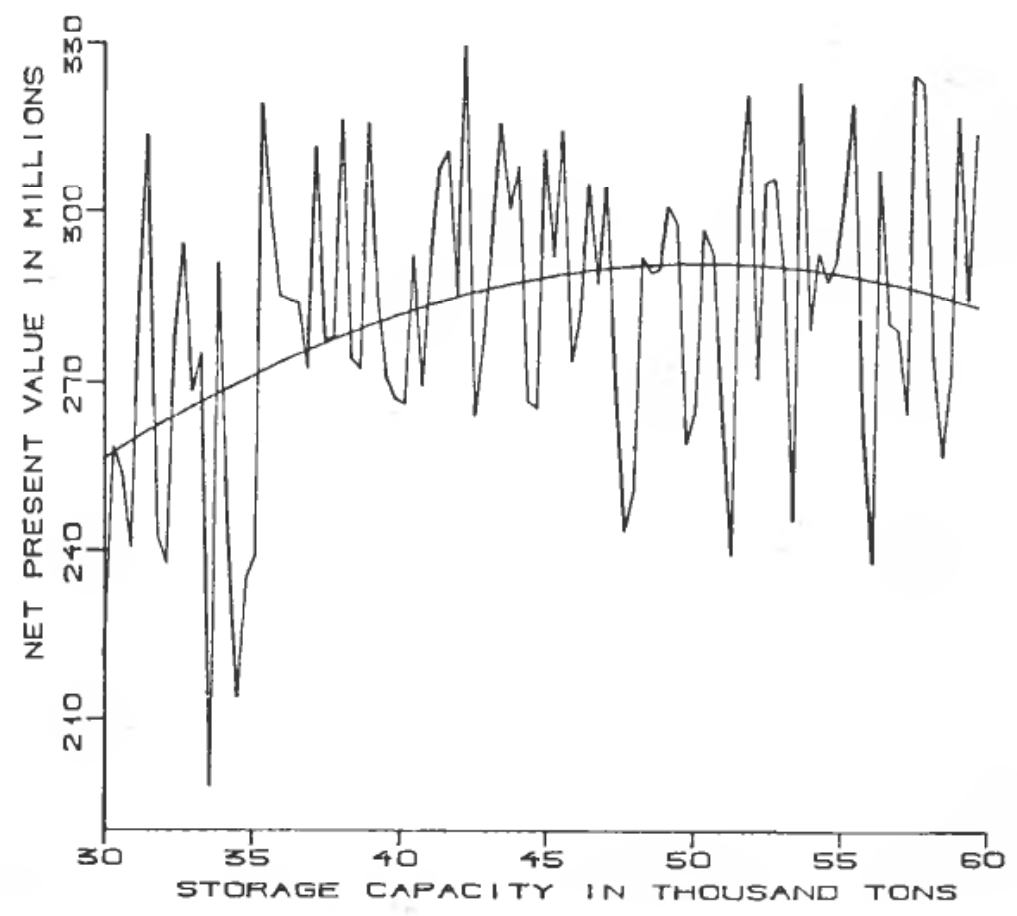

Figure 7. Optimal storage capacity subject to stochastic fluctuations in the net present value. 
Similarly, the profile of the sum of the discounted cash flow shows a steadily increasing tendency towards an asymptote as time goes by. The fluctuations in this curve is reflecting the same stochastic phenomenon which is so much more clear in the storage contents.

The main tendency in the storage contents shows that the frequency of the ship arrivals may be a little too high. There is a slow drifting tendency towards empty storage over the time span of five years. The effect of the emptying storage is also visible in the sum of discounted cash flow, as shown in Fig. 6. This situation may be improved significantly by automatic feed forward adjustment of the shipping plan, as shown later.

Since the storage capacity is a crucial variable, an attempt has been made to optimize this. However, the stochastic nature of the net present value, makes traditional hill-climbing procedures inadequate for finding the optimal storage capacity. Rather a span of capacities is scanned, and the corresponding net present values are smoothed by a second order polynomial in the storage capacity $x$

$$
\mathrm{NPV}=a_{0}+a_{1} x+a_{2} x^{2}
$$

where the maximum is given by the condition

$$
x=a_{t} /\left(2 a_{2}\right)
$$

This situation is shown in Fig. 7. Even though the optimal conditions are hardly visible in the wild fluctuations in the NPV, it is seen that a least squares fit of the second order polynomial gives a reasonable estimation of the best storage capacity.

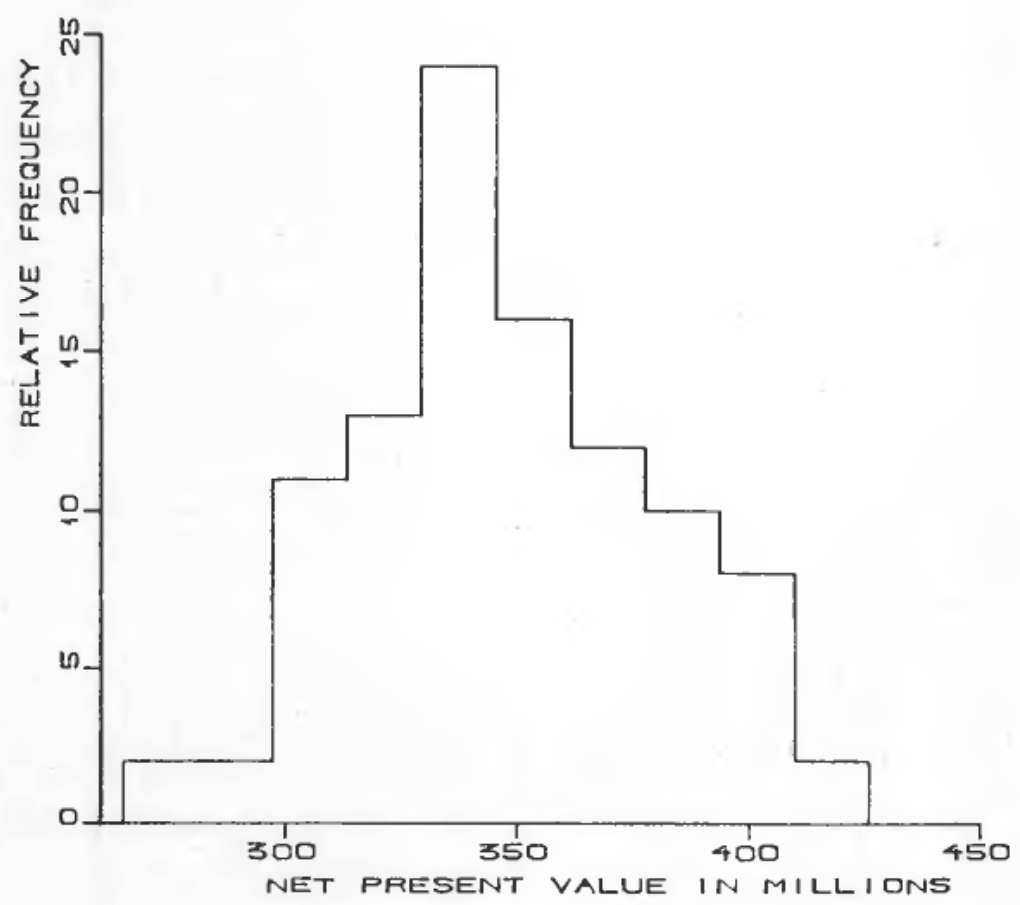

Figure 8. Histogram for computed net present value. 
The stochastic nature of the cash flow results in a probability distribution function for the cash flow in question. This may be illustrated by a series of similar runs but with different starting conditions in the random number generator for the various stochastic events. Such a situation is demonstrated in Fig. 8, where 100 different simulations over a 5 year period are plotted as a frequency histogram for the computed cash flows. The average NPV is 348 million monetary units with a standard deviation of 36.5 million. The highest value computed is 426 million and the lowest 265 million.

This example emphasizes the importance of reliability, random disturbances on the production, the shipping programme, etc. and their effects on the estimated NPV and the standard deviation of the estimate. The example is completely artificial, but illustrates the orders of magnitude of the effects.

\section{The effect of shipping control}

The wild fluctuations in the storage contents and the cash flow profile may be reduced somewhat by a very simple storage control algorithm. This algorithm is of a predictive nature. It is based on the assumption that the long time average of the storage should be one half of full.

At the time of departure of a ship, the most feasible contents of the storage would be the half of the difference between the storage capacity and the average ship capacity. If the contents is below this value, the arrival of the next ship is delayed by an amount which is equal to the time to reach this storage level under normal production

$$
W t=\left(\left(V_{\max }-Q_{0}\right) / 2-Q_{s}\right) / w_{s}
$$

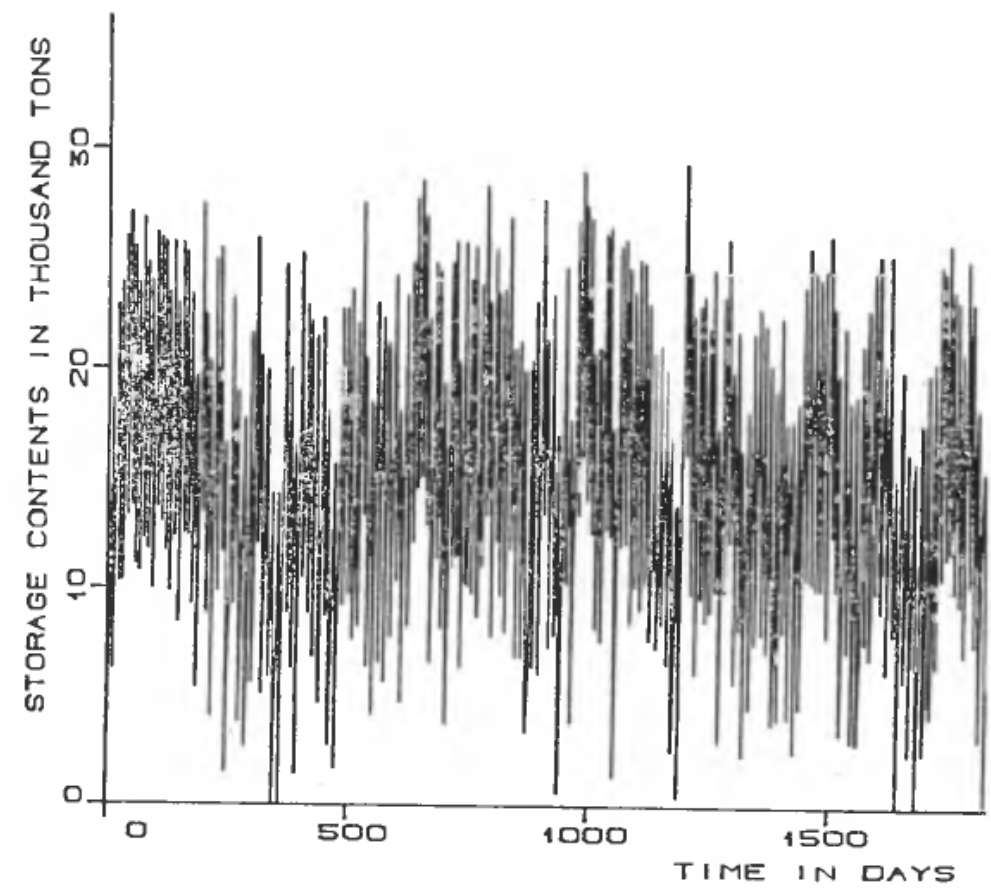

Figure 9. Storage contents over the years, controlled shipping programme. 


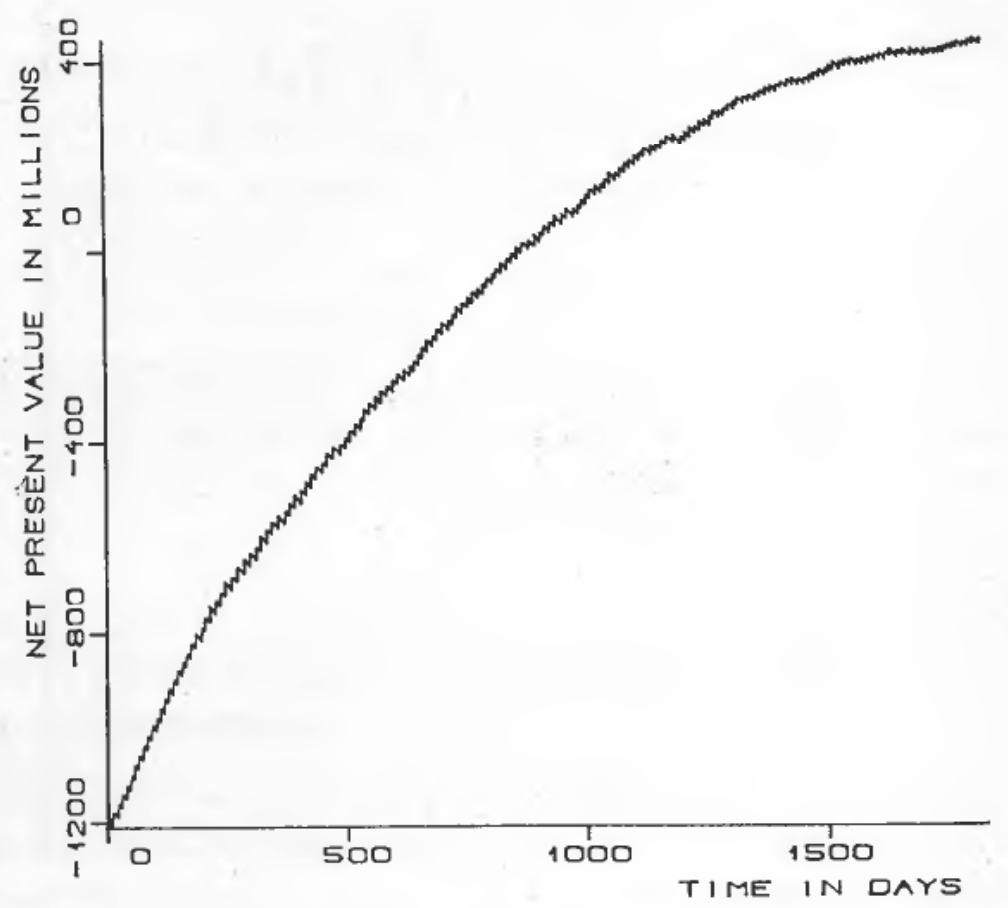

Figure 10. The accumulated discounted cash flow of the invested capital and production.

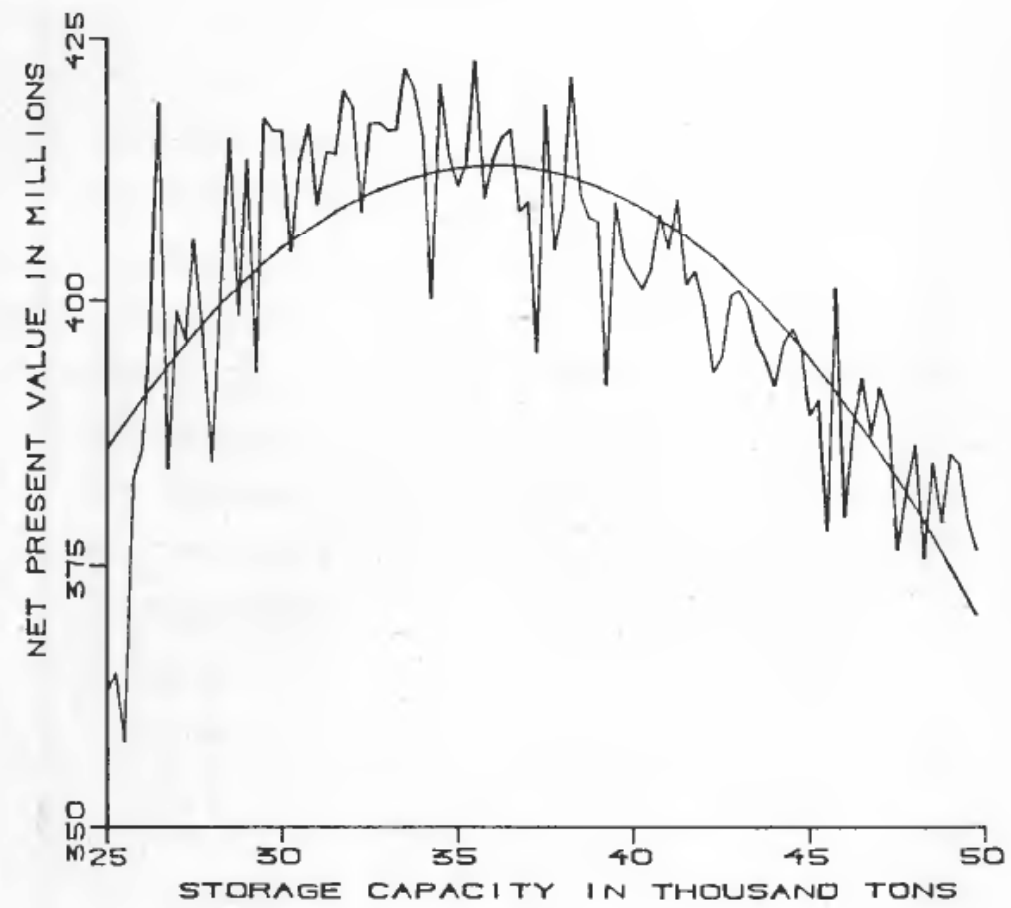

Figure 11. Optimization of storage capacity with prediction control of the shipping programme. 
If this time interval is negative, this is an indication of too much storage remaining in the off-site, and attempts are made to speed up the arrival of the next ship.

It should be emphasized that a predictive control of this nature is not always possible, due to other constraints in the shipping programme. However, the example is useful, as it demonstrates what may be achieved by this kind of shipping control if allowed. Some very special off-shore shipping programmes may satisfy some of the assumptions made here. The control of the shipping programme and not the production rate is based on the assumption that it is desirable to run the plants at maximum capacity.

Figure 9 shows how the storage contents are stabilized towards the middle of the tank by this simple predictive control algorithm. Naturally, the fluctuations in the contents are still significant, duc to the discontinuous export by shiploads from the storage. However, the large, low frequency fluctuations in the uncontrolled case are removed.

The control strategy has a noticeable effect on the net present value, leading to an increase of about $15 \%$. The average NPV now is about 402 million monetary units while the standard deviation is about 58 million. Furthermore, the contents of the storage is stabilized more towards the middle half range of the storage volume, as shown in Fig. 9. The optimal storage capacity is also reduced, as shown in Fig. 11, leading to a lowcr off-site investment.

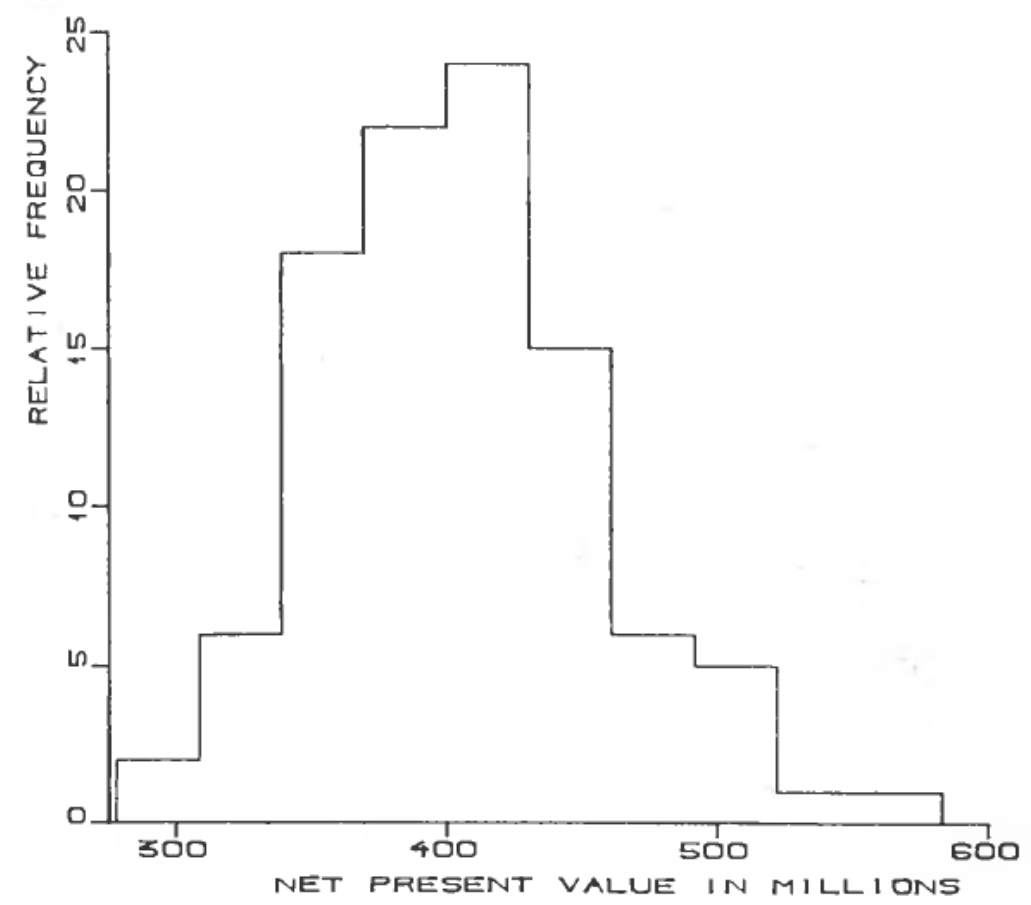

Figure 12. Histogram for computed net present value with predictive control of the shipping programme. 
The corresponding evolution of the net present value is shown in Fig. 10. This curve shows a more steady growth to a higher value over the five year period.

The control algorithm also has an effect on the optimal storage, as shown in Fig. 11. The result is a reduction in the storage capacity of about $28.5 \%$ from 50270 tons to 35946 tons. Hence, the improved NPV may be explained by the two important factors,

A more regular production, i.e. a higher productivity of the plant by a better utilization of the transport system and the storage capacity.

A reduction in the plant investment by reduced off-site storage expenses and investments.

The results of the storage capacity optimization, as explained above, are shown in Fig. 11. It is seen that the random fluctuations are reduced by the simple predictive control algorithm, and that there is a sharper optimum. Similarly the probability distribution function for the NPV is shown in Fig. 12. The estimated average NPV at optimal storage capacity is $\mathbf{4 0 2}$ million monetary units, the maximum computed NPV is 583 million and the minimum 278 million. The standard deviation of the fluctuations in the computation of NPV is 58 million.

\section{REFERENCES}

Asbjørnsen, O. A., and Kreyberg, H. J. (1969). Project evaluation. Computers and Chemical Engineering, 3, 21-27.

Buck, J. R. and HILL, T. W., Jr. (1971). Laplace transforms for the economic analysis of deterministic problems in engineering. The Engineering Economist, 16, 247-263.

Churchicl, R. W. (1958). Operational Mathematics. 2nd Ed. (McGraw-Hill).

JoHNS, W. R., and VARDAS, T. A. (1979). Optimal process design and selection. Computers and Chemical Engineering, 3, 445-454.

TANCHOCO, J. M. A., BuCK, J. R., and LeUNG, L. C. (1981). Modelling and discounting of continuous cash flows under risk. Engng Costs and Prod. Economics, 5, 205-216. 